\title{
Etomidate in the management of severe Cushing's disease and MRSA bacteraemia in a district general hospital in the United Kingdom
}

Stephanie Wei Ping Wong1, Yew Wen Yap', Ram Prakash Narayanan1, Mohammad Al-Jubouri2 , Ashley Grossman ${ }^{3}$, Christina Daousi ${ }^{4}$ and Yahya Mahgoub1

${ }^{1}$ Department of Diabetes and Endocrinology, 2Department of Biochemistry, St Helens and Knowsley Teaching Hospitals NHS Trust, Prescot, UK, ${ }^{3}$ Centre for Endocrinology, William Harvey Research Institute, Barts and London

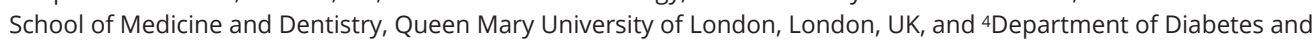
Endocrinology, Aintree University Hospital NHS Foundation Trust, Liverpool, UK

\section{Correspondence} should be addressed to S W P Wong Email

Stephaniewong@doctors. org.uk

\section{Summary}

We report our experience on managing a case of florid Cushing's disease with Methicillin-resistant Staphylococcus aureus (MRSA) sepsis using intravenous etomidate in the intensive care unit of a UK district general hospital.

\section{Learning points:}

- Severe Cushing's syndrome is associated with high morbidity and mortality.

- Etomidate is a safe and effective medical therapy to rapidly lower cortisol levels even in the context of severe sepsis and immunosuppression.

- Etomidate should ideally be administered in an intensive care unit but is still feasible in a district general hospital.

- During treatment with etomidate, accumulation of serum 11 $\beta$-deoxycortisol (11DOC) levels can cross-react with laboratory cortisol measurement leading to falsely elevated serum cortisol levels. For this reason, serum cortisol measurement using a mass spectrometry assay should ideally be used to guide etomidate prescription.

\section{Background}

Patients with Cushing's syndrome may require admission to hospital with a variety of medical emergencies including hypokalaemia, diabetic emergencies, venous thromboembolic events, sepsis, congestive cardiac failure, strokes or myocardial infarction. Management of these conditions can be challenging when the underlying Cushing's syndrome is uncontrolled.

While oral anti-steroidogenesis medications such as ketoconazole and metyrapone are often effective in suppressing excess cortisol levels, this is not an option in certain situations including patients receiving medications through a nasogastric tube necessitating parenteral treatment. In this case report, we describe the use of intravenous etomidate as an effective therapeutic agent to normalise circulating cortisol in a medically decompensated patient until the patient was suitable to switch back to oral cortisol-lowering medications in preparation for hypophysectomy.

\section{Case presentation}

A 54-year-old woman with a past medical history of depression presented to a UK district general hospital with symptomatic hyperglycaemia. Her only regular 
medications were citalopram and olanzapine. On admission, recorded capillary blood glucose (CBG) level was $30 \mathrm{mmol} / \mathrm{L}$ with a normal $\mathrm{pH}$ of 7.49 . Physical examination demonstrated truncal obesity, proximal muscle weakness, skin bruises and elevated blood pressure $(163 / 114 \mathrm{mmHg})$, raising the suspicion of Cushing's syndrome. There was also an incidental finding of a large right-sided haematoma on the posterior aspect of her thorax.

\section{Investigation}

Initial investigations identified a low potassium levels of $2.3 \mathrm{mmol} / \mathrm{L}$, a markedly elevated random cortisol of $2070 \mathrm{nmol} / \mathrm{L}$ (reference range 145-619), and plasma adrenocorticotrophic hormone (ACTH) of $125 \mathrm{pmol} / \mathrm{L}$ (reference range 2-11). Glycated haemoglobin (HbA1c) was high at $115 \mathrm{mmol} / \mathrm{mol}$. The rest of the baseline pituitary hormone profile was within the normal reference range. An autoimmune screen for diabetes (glutamic acid decarboxylase and islet cell antibodies) was negative. Serum cortisol levels remained elevated (>50 nmol/L) following standard overnight, 48-h lowdose and high-dose dexamethasone suppression tests. Magnetic resonance imaging of the pituitary identified a $16 \times 16 \times 18 \mathrm{~mm}$ hypoenhancing lesion arising from the right half of the pituitary gland (Figure 1). Computed tomography scans of the chest, abdomen and pelvis did not identify any potential ectopic ACTH sources. These results were consistent with a diagnosis of Cushing's disease arising from a large pituitary macroadenoma.

\section{Treatment}

Initial management involved treating her metabolic abnormalities. She was commenced on twice-daily biphasic insulin therapy. Potassium levels were treated with potassium replacement (oral and intravenous) and hypertension managed with spironolactone and ramipril. Treatment for Cushing's syndrome was initiated with oral metyrapone $250 \mathrm{mg}$ three times daily. Serum cortisol levels improved to a nadir of $900 \mathrm{nmol} / \mathrm{L}$. The patient was managed on a general medical ward with periods of coronary care input for the management of hypokalaemia.

Her clinical condition deteriorated due to severe cellulitis secondary to infected haematoma of the chest wall. The infected haematoma was surgically drained and she was subsequently treated with intravenous vancomycin after a wound swab culture was positive for Methicillin-resistant Staphylococcus aureus (MRSA).
Around a week after metyrapone was commenced, she unfortunately developed hospital-acquired pneumonia complicated by acute respiratory distress syndrome (ARDS). The level of hypoxia necessitated transfer to intensive care unit for intubation and ventilation. A nasogastric tube was inserted for medications and feeds. However, neither metyrapone capsules nor crushed ketoconazole tablets are reliably absorbed through a nasogastric tube. As a consequence, the serum cortisol levels peaked to $3319 \mathrm{nmol} / \mathrm{L}$. A surgical option of suppressing serum cortisol by
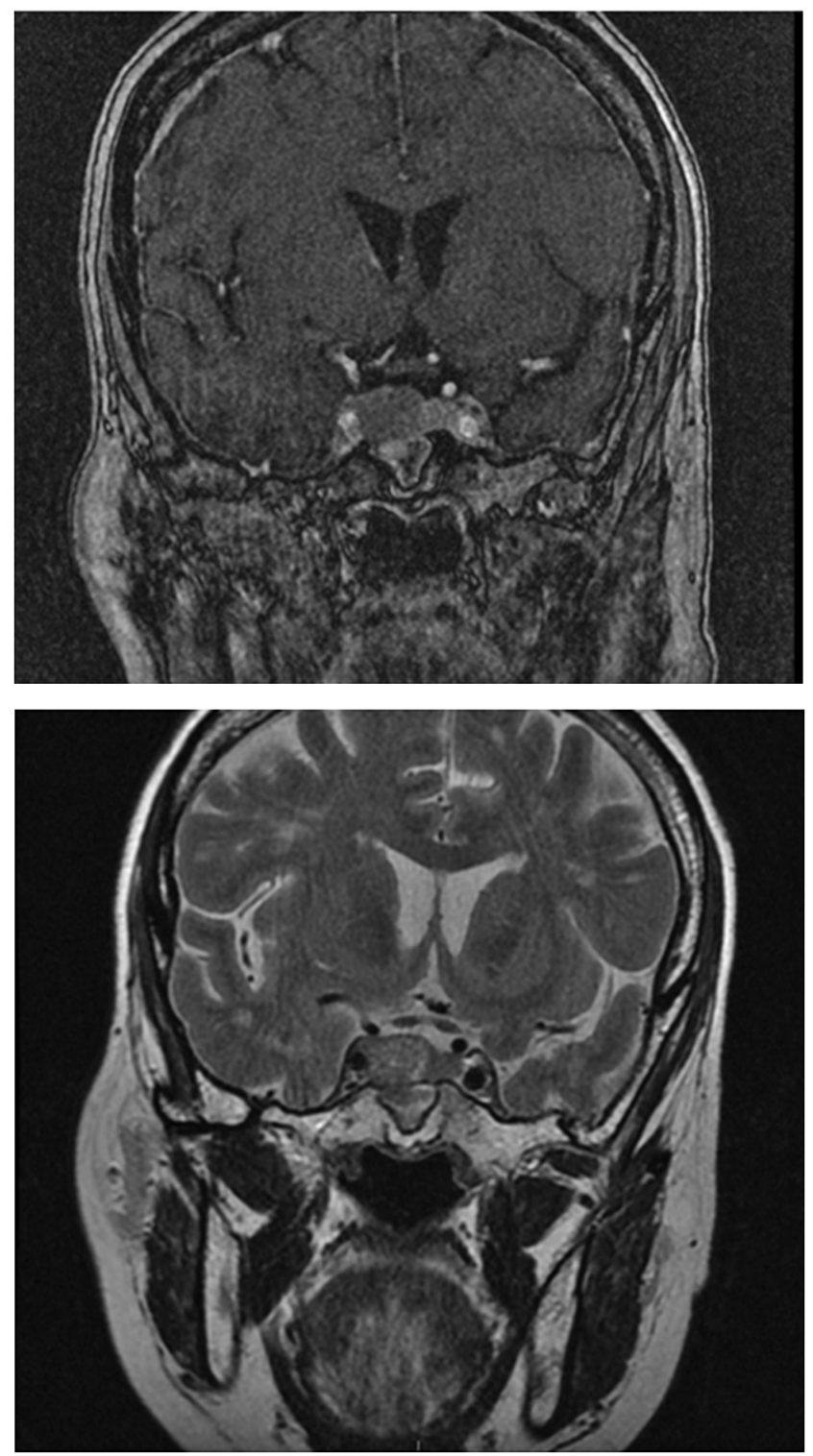

\section{Figure 1}

Coronal view of MRI pituitary showing a right-sided pituitary macroadenoma on T1 (upper figure) and T2 (lower figure) weighted images. 
Table 1 Serum cortisol levels during the etomidate infusion in hours.

\begin{tabular}{|c|c|c|c|c|c|c|c|c|c|c|c|c|c|c|}
\hline & \multicolumn{14}{|c|}{ Time (h) } \\
\hline & 0 & 4 & 8 & 12 & 16 & 20 & 24 & 28 & 32 & 36 & 40 & 72 & 96 & 120 \\
\hline Cortisol level (nmol/L) & & & & & & & & & & & & & & \\
\hline Local lab & 3319 & 1735 & 1546 & 1467 & 1140 & 1224 & 1203 & 1236 & 1079 & 1112 & 1035 & - & - & - \\
\hline Mass spectrometry & - & - & - & - & - & 726 & 766 & 701 & 620 & 734 & 702 & 634 & 463 & 463 \\
\hline
\end{tabular}

undergoing a bilateral adrenalectomy was unsafe given the degree of immunosuppression, the state of metabolic decompensation and severe sepsis.

Parenteral etomidate was trialled under close supervision in the intensive care unit as neither oral nor surgical options were viable options. Etomidate was commenced at $1.25 \mathrm{mg} / \mathrm{h}$ and then titrated up to $2.5 \mathrm{mg} / \mathrm{h}$. Cortisol was measured every $4 \mathrm{~h}$ to achieve a target level of between 600 and $800 \mathrm{nmol} / \mathrm{L}$; higher serum cortisol level was aimed to reach a physiological level allowing for a stress response for sepsis. Once serum cortisol levels were below $600 \mathrm{nmol} / \mathrm{L}$, treatment was switched to block-and-replace regime using intravenous hydrocortisone infusion at $1 \mathrm{mg} / \mathrm{h}$ in addition to etomidate.

Table 1 and Figure 2 charts the serum cortisol measurements while on etomidate infusion measured using two different cortisol assays. Due to crossmeasurement of 11 $\beta$-deoxycortisol (11DOC) in our local hospital assay (Siemens Centour XP), a more reliable cortisol assay using mass spectrometry (to avoid 11DOC cross-reactivity) from a hospital laboratory in Manchester was used for subsequent cortisol measurements.

\section{Outcome and follow-up}

On Day 2 of the etomidate infusion, she was extubated without difficulty. There was a resolution of the hypokalaemia as a result of the lower serum cortisol levels. Markers of inflammation (C-reactive protein and white cell count) and insulin requirements began to

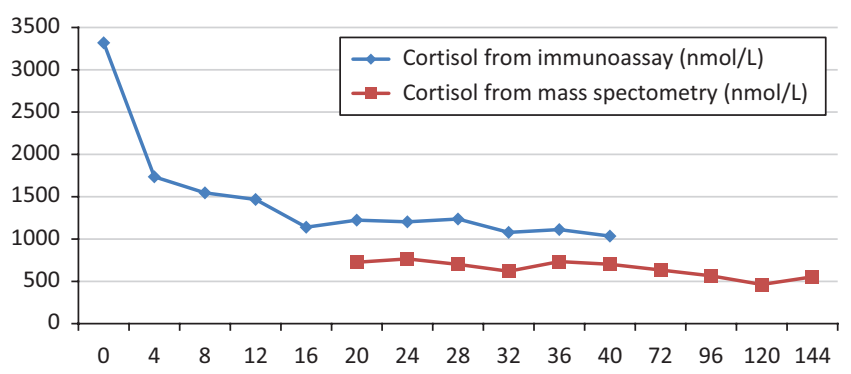

Figure 2

Serum cortisol value during the etomidate infusion in hours. decrease. The infusion was maintained for another 6 days until she was transferred to a tertiary care endocrine centre for surgery. Later, she underwent transsphenoidal hypophysectomy for resection of the Cushing's disease tumour and made a good recovery. Immediate postoperative serum cortisol levels were less than $1000 \mathrm{nmol} / \mathrm{L}$ and she was commenced on prophylactic intravenous hydrocortisone. Subsequently, she did not require further anti-steroidogenesis or insulin treatment. Resected pituitary histology confirmed the diagnosis of Cushing's disease where it stained positive for adrenocorticotrophic cells with Ki-67 index less than 3\%. She has remained in remission 1 year postsurgical resection and continued on oral hydrocortisone. In the following months after surgery, serial overnight dexamethasone suppression test showed appropriate suppression of serum cortisol levels to than $50 \mathrm{nmol} / \mathrm{L}$, while short Synacthen test demonstrated adequate adrenal reserves enabling gradual down-titration of oral hydrocortisone. However, such early return of pituitary-adrenal function suggests that recurrence is likely.

\section{Discussion}

This case firstly illustrates the importance of investigating for Cushing's syndrome when patients present with clinical or biochemical features which are compatible with this condition and treating the metabolic alterations as a matter of urgency.

There is a range of medical pharmacological options for the management of patients with Cushing's syndrome, including ketoconazole, metyrapone and etomidate.

Ketoconazole is an imidazole derivative that works as a potent inhibitor of both cortisol and aldosterone synthesis by inhibiting the $17 \alpha$-hydroxylase, 11-hydroxylation steps of steroid biosynthesis and at higher doses also blocks the cholesterol side-chain cleavage enzyme (1) Endocrine Society Guidelines suggest that oral ketoconazole should be dosed at 400-1600 mg per day (2). Cortisol levels and liver function tests are required at baseline and monitored on treatment initiation to establish a suitable effective dose (1). 
Metyrapone works at the site of the adrenal cortex by being a competitive inhibitor of $11 \beta$-hydroxylation (1). This results in the inhibition of cortisol production, leading to an increase in ACTH production which leads to an increased synthesis of cortisol precursors (1). This drug has a rapid onset of action and can be dosed as an oral capsule at $500 \mathrm{mg}$ to $6 \mathrm{~g}$ per day. Metrypone dosage can be dose titrated according to cortisol levels or alternatively can be prescribed to block cortisol production altogether in combination with exogenous corticosteroid replacement as a 'block-and-replace' therapy.

Etomidate is currently the only pharmacological agent which can is given intravenously. It has a rapid time of onset and generally requires monitoring in an intensive care unit (2). Etomidate is the drug of choice for acutely unwell patients with severe hypercortisolism who are not medically safe for imminent surgery (2). This can be considered as a bridging therapy to other medical or surgical treatment (3). It works by inhibiting

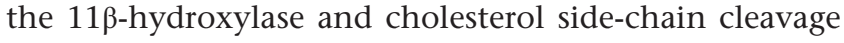
and rapidly decreases steroidogenesis within $12-24 \mathrm{~h}$ (3). A loading dose of $3-5 \mathrm{mg}$ is followed by a continuous infusion of $0.03-0.10 \mathrm{mg} / \mathrm{kg} / \mathrm{h}(2.5-3.0 \mathrm{mg} / \mathrm{h})$ for partial blockade $(2,4)$. Continued monitoring of cortisol levels every $4-6 \mathrm{~h}$ is recommended with titration of the infusion rates, with the aim to establish a stable serum cortisol level between $280-560 \mathrm{nmol} / \mathrm{L}(10-20 \mu \mathrm{g} / \mathrm{dL})$ either on its own or as a block-and-replace strategy (2). Preda et al. suggested a target serum cortisol of $500-800 \mathrm{nmol} / \mathrm{L}$ in a physiologically stressed patient and a circulating cortisol range of $150-300 \mathrm{nmol} / \mathrm{L}$ in a non-physiologically stressed patient (5). Potential adverse events include apnoea, hyperventilation, nausea and vomiting, stridor and arrhythmias (1).

Etomidate was used historically as an anaesthesia induction agent (2) until a study in 1983 reported increased mortality rates among intensive care patients receiving prolonged etomidate infusions for sedation, in comparison to patients receiving benzodiazepines $(5,6)$. Of note, another study reported that in a similar group of critically ill patients receiving etomidate infusions, cortisol replacement therapy was associated with a lower mortality rate (7).

For patients who are acutely unwell with sepsis and in an immunocompromised state, uncontrolled Cushing's syndrome can be life threatening. As reported in our case, the administration of oral medications to treat this condition may not be an option in critical care. Etomidate offers a reliable means of cortisol lowering in this situation through an intravenous administration route, but requires supervision, generally in a critical care setting. In a review article by Preda et al., it was noted that in the case reports that had been reviewed, most of the patient had normalised their cortisol levels with the use of etomidate infusions. The duration for which etomidate was continued ranged between $5 \mathrm{~h}$ and 5.5 months. The authors also recommended that these patients are managed in intensive care or high dependency settings (4).

Over the last few years, etomidate infusions have also been administered in general medical ward settings. Soh et al. report a protocol for the treatment of Cushing's syndrome without the need for admission to an intensive care unit (8). Their protocol utilises a starting dose of $2.5 \mathrm{mg} / \mathrm{h}$, regardless of body weight, to be titrated according to cortisol levels, up to a maximum of $4 \mathrm{mg} / \mathrm{h}$. Whenever the cortisol levels of patients drop to less than $200 \mathrm{nmol} / \mathrm{L}$, the additional hydrocortisone therapy is initiated as a form of 'block-and-replace' treatment regime. They reported successfully treating four patients with severe hypercortisolism from Cushing's disease with etomidate in a general medical ward setting.

We would recommend that the use of etomidate in a ward setting should be restricted to hospitals where ward staff are familiar with the drug and its monitoring and in patients not clinically compromised or requiring significant organ support. Communication with the local intensive care team would be recommended as these patients can have the potential to deteriorate systematically. Nonetheless, reports of administering this drug in a medical ward setting when this can be safely undertaken offers further opportunities for reliable cortisol control in patients not adequately controlled on oral medications, and in our case can be performed in a general medical hospital under intensive care supervision.

It has been observed that the serum cortisol levels measured using immunoassay in our clinical laboratory were elevated compared to mass spectrometry. This may reflect the mechanism of action of etomidate on the steroidogenesis pathway resulting in the accumulation of 11DOC. Additionally, the discrepancy between immunoassay and mass spectrometry in our case highlights that assay interference can occur on different anti-steroidogenesis medications (9). Thus, it is important to utilise and consider mass spectrometry in the measurement of serum cortisol samples in patients on pharmacological therapy for Cushing's syndrome. 
In conclusion, we demonstrate that etomidate can be successfully used in a general acute hospital setting in a patient with severe Cushing's syndrome and MRSA bacteraemia, and show that, as for etomidate, the cortisol assay must be considered in terms of cross-reactivity with 11DOC.

Declaration of interest

The authors declare that there is no conflict of interest that could be perceived as prejudicing the impartiality of the research reported.

\section{Funding}

This research did not receive any specific grant from any funding agency in the public, commercial or not-for-profit sector.

\section{Patient consent}

Written consent was obtained from the patient for publication of the submitted article and accompanying images.

\section{Author contribution statement}

$S W$ and $Y W Y$ wrote the report. $Y W Y, Y M, C D, P N$ contributed to the diagnosis and management of the patient. $\mathrm{M} J$ advised on the laboratory levels of serum cortisol. A B G advised on the use of etomidate.

\section{References}

1 Joint Formulary Committee. British National Formulary. London: BMJ Group and Pharmaceutical Press, 2017.

2 Nieman LK, Biller BMK, Findling JW, Murad MH, Newell-Price J, Savage MO \& Tabarin A. Treatment of Cushing's syndrome: an Endocrine Society clinical practice guideline. Journal of Clinical Endocrinology and Metabolism 2015100 2807-2831. (https://doi. org/10.1210/jc.2015-1818)

3 Schulte HM, Benker G, Reinwein D, Sippell WG \& Allolio B. Infusion of low dose etomidate: correction of hypercortisolemia in patients with Cushing's syndrome and dose-response relationship in normal subjects. Journal of Clinical Endocrinology and Metabolism 199070 1426-1430. (https://doi.org/10.1210/jcem-70-5-1426)

4 Preda VA, Sen J, Karavitaki N \& Grossman AB. Etomidate in the management of hypercortisolaemia in Cushing's syndrome: a review. European Journal of Endocrinology 2012167 137-143. (https://doi. org/10.1530/EJE-12-0274)

5 Ledingham IM \& Watt I. Influence of sedation on mortality in critically ill multiple trauma patients. Lancet 19831 1270. (https:// doi.org/10.1016/s0140-6736(83)92712-5)

6 Watt I \& Ledingham IM. Mortality amongst multiple trauma patients admitted to an intensive therapy unit. Anaesthesia 198439 973-981. (https://doi.org/10.1111/j.1365-2044.1984.tb08885.x)

7 Wei F \& McKee JI. Serum cortisol levels in severely stressed patients. Lancet 19821 1414. (https://doi.org/10.1016/S0140-6736(82)92531-4)

8 Soh LM, Gunganah K, Akker SA, Jones P, Khachi H, Dodzo K $\&$ Drake WM. Etomidate in the emergency management of hypercortisolemia. European Journal of Endocrinology 2012167 727-728; author reply 729. (https://doi.org/10.1530/EJE-12-0698)

9 Owen LJ, Halsall DJ \& Keevil BG. Cortisol measurement in patients receiving metyrapone therapy. Annals of Clinical Biochemistry 2010 47 573-575. (https://doi.org/10.1258/acb.2010.010167)

Received in final form 27 July 2019

Accepted 20 August 2019 